\title{
Power Balance and Control in Optically Controlled Phased Array Antenna, Based on Optical True Time Delay
}

\author{
Ling Zhou ${ }^{1}$, Xiang-wen $\mathrm{Li}^{1}$, Shuang Zhang ${ }^{1}$ and Yi-he Liu ${ }^{*}, 2$ \\ ${ }^{I}$ The Engineering \& Technical College of Chengdu University of Technology, Leshan, 614000, P.R. China \\ ${ }^{2}$ College of Computer Science, Neijiang Normal University, Neijiang, 641000, P.R. China
}

\begin{abstract}
There is clear difference in each fiber-optic link's power output, in the large scale optically controlled phased array antenna. In order to realize the optical power balance and control between the various channels, the equilibrium or control power of each optical signal needs to be considered, and it will reduce the optical beam forming network generated side-lobe beam, which results in the performance improvement of the system. In this paper, the methods of optical power equalization and control have been proposed, which is used in the forming network of the optical beam. The method can realize the optical power's equalization and control of each optical signal. In the end of this paper, the verification and results are provided.
\end{abstract}

Keywords: Optically controlled phased array radar (ocpar), Fiber-optic link, Optical true time delay (OTTD), Optical power, Power balance and control.

\section{INTRODUCTION}

Some researchers advanced a new theory relatively early, which is using optical fiber as delay medium in the signal processing of microwave and millimeter waves. The technology of Optical True Time-Delay is appeared, and it is the earliest application, which is used in microwave photonic filter and optically controlled phased array antenna. The technological idea of OCPAA, which is based on OTTD, is as follows: the electrical signal, with its frequency greatly lower than the optical frequency, can be loaded into the optical wave. Then the loaded optical wave can be delayed, and the electrical signal can be extracted from it by optical detector. The extracted electrical signal and the original electrical one have identical characters, but there are some time-delay on phase position between the two signals. The True Time Delay(TTD) system based on this technological process is called the OTTD system.

The integration of OTTD and phased array antenna technique has overcome two technical bottlenecks in the conventional phased array radar, i.e., the restriction of large instantaneous bandwidth and the beam deflection. The pure electrical real time delay unit has been replaced by the optical real time delay unit in optically controlled phased array antenna system, which makes the new OCPAA system have the virtue of low cost, light weight, small size, low power consumption and strong capability of anti-electromagnetic interference. In addition, OTTD has extensive application in optical switching technique and wireless communication technology.

*Address correspondence to this author at the College of Computer Science, Neijiang Normal University, Neijiang, 641000, P.R. China; Tel: +86 832 2343466; Fax: +86 832 2340320; E-mail: iu_yihe@163.com
Compared to conventional electronic beam forming network, the optical beam forming network based on OTTD has many advantages, such as low power consumption, small size, large bandwidth, anti-electromagnetic interference and effective restriction of beam deflection, etc. Thus, many OTTD systematic configuration models have been invented. However, after experiments, due to various factors, e.g. large amount of photovoltaic components and parts in optical beam forming network with its inconsistent wavelengths response, the gain difference of diverse wavelength signals caused by Erbium-Doped Fiber Amplifier (the gain unevenness), the inconsistent insertion loss of filters at different wavelength positions, as well as the inconsistent photoelectric detectors response, the optical power input in photoelectric detectors through channels are not equal. The optical power unequalization results in the functional deterioration of microwave in space distribution, for example, the low main-lobe peak, the high sidelobe level, and the accuracy decrease of beam-pointing. As a result, it is necessary to precisely control the optical signal power in each channel for the smooth operation of optical beam forming network.

\section{MAJOR FACTORS OF OPTICAL BEAM FORM- ING NETWORK}

\subsection{The Gain Unevenness of Erbium-Doped Fiber Amplifier}

Erbium-Doped Fiber Amplifier, in the range of 1530$1560 \mathrm{~nm}$, consists of Erbium-doped fiber, one or a plurality of pump laser, passive wavelength coupler, and optical isolator or tap coupler. Generally speaking, pump light and signal light can be input into optical amplifier along the same 


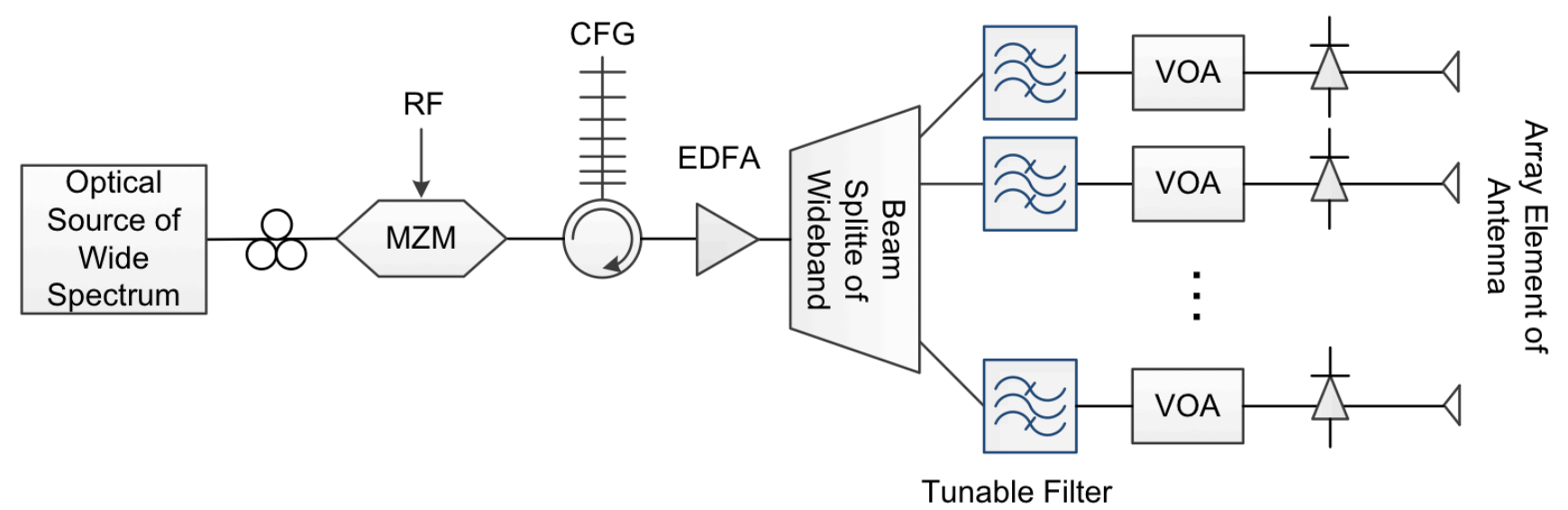

Fig. (1). The power control in optical beam forming network.

direction, or the reverse direction, as well as both directions simultaneously. Normally, the $980 \mathrm{~nm}$ pump wavelength is a top priority because of its relatively low noise and large particle number inversion. Along with the increase of the EDFA input signal power, amplifier gain first maintains the small signal gain, then decreases to gain saturation zone when a linear decrease occurs. The gain of $0 \mathrm{~dB}$ can be obtained as long as the EDFA input signal power is strong enough. Although the EDFA can amplify every wavelength, the gain tilt is doomed to occur because the photon energy is related to the signal wavelength.

\subsection{Inconsistent Responsiveness of Photoelectric Detec- tor}

The responsiveness of photoelectric detector is influenced by the wavelength and Photoelectric diode material. For a given material, the bigger the wavelength of the incident light, the smaller the photon energy. When the energy is too small to meet the requirement of igniting an electron to transmit to the conduction band, the responsiveness sharply descends at the cutoff wavelength point. It is particularly necessary for the relatively high request for the photoelectric detector's responsiveness consistency in optical beam forming network, so that the PD array consisting of large amount of photoelectric detectors is required to detect the wide-range wavelength of the optical signal.

\subsection{The Stability of Optical Source}

The threshold current increases along with the rising temperature in semiconductor laser, and the stimulated radiation threshold varies with the increase of laser service life. The interaction of all factors is extremely complicated. Therefore, the DC bias current is bound to adjust if the output optical power of the laser needs to maintain stably. It might require large amount of lasers in optical beam forming network, some wavelength tunable lasers, and others specified output wavelength lasers. How to guarantee the consistency and stability of these lasers is another important problem to think over.

\subsection{Power Balance and Control}

Through the above analysis, it is known that the interaction of multiple factors leads to the power unequalization in all channels in optical beam forming network. In order to obtain the smooth operation of the network, it is especially important to achieve the precise control signal power. In application, a suitable dynamic power equalization module should be added to achieve the equal signal power of RF in all channels. The dynamic power equalization is used to reduce the attenuation of certain wavelengths and balance them. Based on the feedback information by the monitor, the parameters can be adjusted to fit the desired channel specification so as to flexibly adapt to the optical power fluctuation caused by the changes of the network. Furthermore, based on the dynamic power equalization, the precise power control can be achieved, that is to say, weighting function can be added in to control the electromagnetic wave sidelobe, thus more effectively enhance the function of optical beam forming network. Due to the inconsistent wavelength response of optical devices in optical beam forming network, the gain unevenness of diverse wavelength signal that is caused by EDFA, the inconsistent insertion loss of filters at different wavelength position, and the inconsistent responsiveness of PD, etc. the performance of the system can degrade sharply. The following example, a beam-forming network based on the wide spectrum light source and chirped fiber grating, illustrates the above point, shown as the typical structure in the Fig. (1): The output light from wide spectrum optical source is first modulated by radio - frequency signal in electro-optical modulator, and enters the chirped fiber grating (CFG) through the circulator, and then, after EDFA amplification, the light was divided into $\mathrm{N}$ channels. When passing through a dispersive element (CFG), the light signals with different wavelengths produce different time delay, which finally produce differential delay caused by the different tunable filters matching to different-wavelength light signals in every channel. Thus the different beam steering can be achieved. The Variable Optical Attenuator (VOA) is added here in order to achieve the dynamic optical signal level control. And the precise control of optical power can be 
Table 1. Weight coefficients and relative optical powers of 8 element linear array.

\begin{tabular}{|c|c|c|c|c|c|c|c|c|}
\hline Channel & 1 & 2 & 3 & 4 & 5 & 6 & 7 & 8 \\
\hline Normalized Weight & 0.210 & 0.5137 & 0.8163 & 1 & 1 & 0.8136 & 0.5137 & 0.210 \\
\hline
\end{tabular}

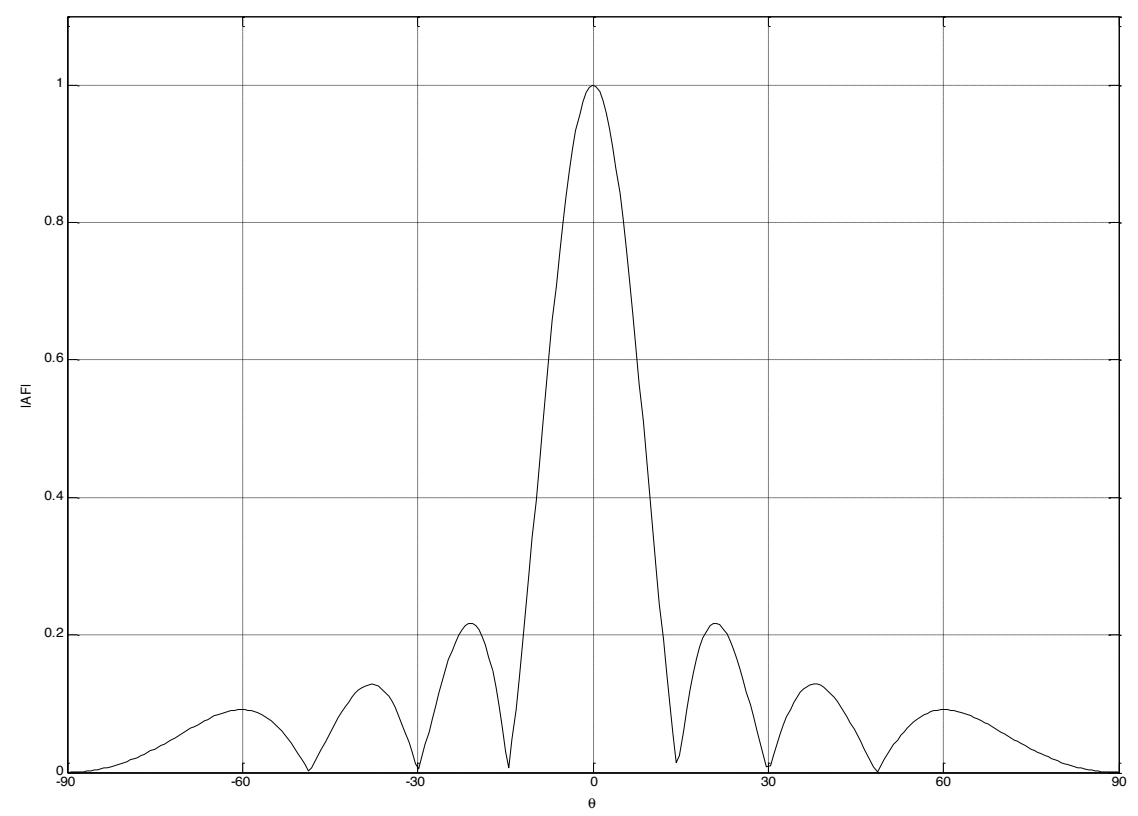

Fig. (2). The Array Factors under the Condition of Power Equalization.

obtained by attenuation of optical power through the operation of machinery, calorescence, MEMS or electro-optical technology, without affecting other characters of the optical signals.

Under the condition of optical power equalization in the system, that is, all isotropic matrix elements in the optical beam forming network have the equal amplitude, and the array factor can be calculated from formula 1 :

$A F_{n}=\sin \left[\frac{N k d}{2}\left(\sin \theta-\sin \theta_{0}\right)\right] / N \sin \left[\frac{k d}{2}\left(\sin \theta-\sin \theta_{0}\right)\right]$

In this formula, $\mathrm{k}$ is wave numbered, is array element spacing, $\mathrm{N}$ is the number of array element. The array factors have obvious sidelobes when $\theta_{0}=0$, as shown in Fig. (2). The sidelobe means that the antenna array radiates some energy toward unexpected direction. The above problem can be restrained by the method of dynamic power control.

By means of Kaiser-Bessel weighting, it can effectively restrain sidelobe level through Controlling the optical power weighted of each delay optical channel, which is shown in formula 2:

$$
w(k)=I_{0}\left[\pi \alpha \sqrt{1-(2 k / N)^{2}}\right] / I_{0}[\pi \alpha], k=0,1, \mathrm{~L}, N / 2, \alpha>1
$$

Under the condition of $\alpha=3$ and $\mathrm{N}=8$, the weight of Kaiser-Bessel and relative powers of the linear array, is composed of eight arrays, as shown in Table 1. Compared to Fig. (2), Fig. (3) displays the array factors after the weighting control, which can clearly show that the sidelobe level can obtain $0 \mathrm{~dB}$ in the antenna array with Kaiser-Bessel weighting, but the antenna array maintains the same beam width as in uniformity weighting (as shown Fig. 2). Therefore, the technology of dynamic optical power control can effectively restrain the sidelobe level generated by forming network of optical beam, and further improve the performance of optical beam forming network.

\section{CONCLUSION}

In conclusion, the sidelobe, generated by forming network of optical beam, can be effectively restrained by the dynamic optical power control. As shown in the simulation results, the antenna sidelobe level with Kaiser-Bessel weights becomes zero, while the beam width almost remains at the same level. So, the application of the dynamic optical power control can effectively improve further the performance of the forming network of optical beam. 


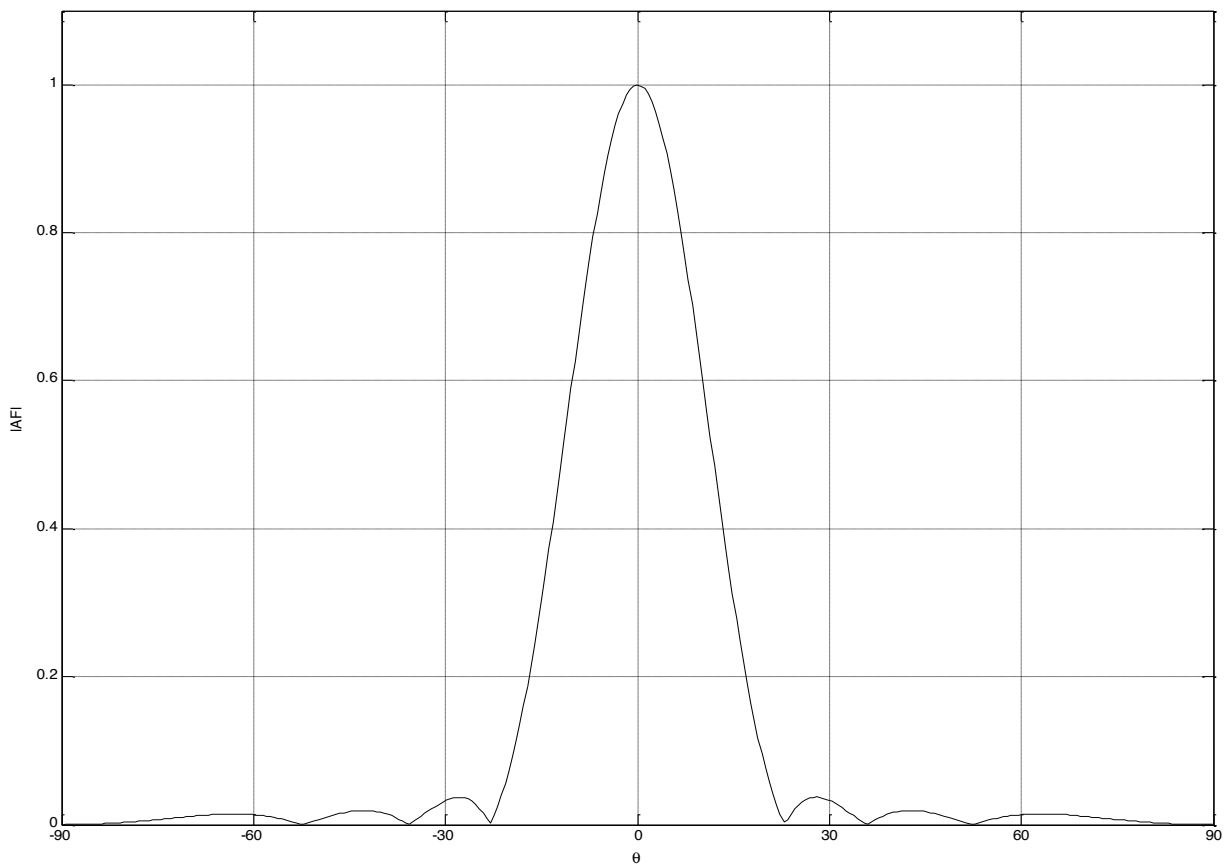

Fig. (3). The array factor by Kaiser-Bessel weighting under the dynamic optical power control.

\section{CONFLICT OF INTEREST}

The author confirms that this article content has no conflict of interest.

\section{ACKNOWLEDGEMENTS}

This work is supported by The Key Fund Project of Sichuan Provincial Department of Education under Grant13ZA0003, Grant 14ZB0360, Grant 14ZB0363, Grant 14ZB0352; The Sichuan Province Department of Science and Technology under Grant 2015JY0119; the Science and Technology Development Fund of Macau (FDCT) under Grant 014/2007/A1, Grant 063/2009/A, and Grant 024/2009/A1; the Research Committee of the University of Macau, under Grant RG072/09-10S/MPU/FST; the Key Fund Project of Leshan Science and Technology Bureau (14GZD046).

\section{REFERENCES}

[1] J. Tong, J. K. Wade, D. L. MacFarlane, and H. Shi, "Active integrated photonic true time delay device," IEEE Photon. Technol. Lett., vol. 18, no.16, pp. 1720-1722, 2006.

[2] Z, Shi, L. L. Gu, Y. Q. Jiang, Y. Q. Jiang, and R. T. Chen, "Truetime-delay module based on a single tunable laser in conjunction with a waveguide hologram for phased array antenna application," Optical Engineering, vol. 44, no. 8, pp. 084301-1-084301-7, 2005.

[3] Y. Chen, and $\mathrm{K}$. Wu, "Reconfigurable true-time delay for wide band based-array antennas," Proc. of SPIE, vol. 5363, pp. 125-130, 2004.

[4] J. J. Lee, R. Y. Loo, S. Livingston, and V.I. Jones, "Photonic wideband array antennas," IEEE Transactions on Antennas and Propagation, vol. 43, no. 9, pp. 966-982, 1995.

[5] B. M. Jung, and J. D. Shin, "Optical true time-delay for twodimensional X-band phased array antennas," IEEE Photon. Technol. Lett., vol. 19, no. 12, pp. 877-879, 2007.

[6] S. Yuan, "Photonic delay line architecture using MEMS based photonic switch,' Proceedings of SPIE The International Society for Optical Engineering, vol. 5201, pp. 23-33, 2003.

[7] W. D. Jemison, and P. R. Herczfeld, "Acousto-optically controlled true time delays-experimental results," IEEE Microwave and Guided Wave Letters, vol. 6, no. 8, pp. 283-285, 1996.

[8] J. G. Willms, A. Ouacha, L. D. Boer, and F.E. van Vliet, "A wideband GaAs 6-bit true-time delay MMIC employing on-chip digital drivers," TNO Physics and Electronics Laboratory, 2000. [www.tno.n1/instit/fel/os/resources/GAAS2000_6bit_TTD.pdf].

[9] D. Dolfi, P. Joffre, J. P. Huignard, D. Philippet and P. Granger, "Experimental demonstration of a phased-array antenna optically controlled with phase and time delays," Applied Optics, vol. 35, no. 9, pp. 5293-5300, 1996.

[10] D. A. Henderson, C. Hoffman, R. Culhane, and D. III Viggiano, "Kilohertz scanning, all-fiber optical delay line using piezoelectric actuation," In: Proceedings of SPIE The International Society for Optical Engineering, vol. 5589, pp. 99-106, 2004. 